\title{
Tissue responses to ischemia
}

\section{SERIES INTRODUCTION \\ Tissue ischemia: pathophysiology and therapeutics}

\author{
Gregg L. Semenza \\ Institute of Genetic Medicine, The Johns Hopkins University School of Medicine, CMSC-1004, 600 North Wolfe Street, \\ Baltimore, Maryland 21287-3914, USA. Phone: (410) 955-1619; Fax: (410) 955-0484; E-mail: gsemenza@jhmi.edu.
}

This issue of the JCI contains the first articles in a Perspective series that focuses on ischemia, the major cause of mortality in the developed world. The specific mechanisms and consequences of ischemia differ in each tissue or organ, which reflects differences in anatomy and physiology. For this reason, the series has been organized to include articles on cerebral (Dennis Choi and colleagues), myocardial (Sandy Williams and Ivor Benjamin), and skeletal muscle (Jeff Isner) ischemia, as well as discussions of ischemia in epithelial tissues (Sanjay Nigam and colleagues) and hypoxia-induced pulmonary vascular remodeling (Norbert Voelkel and Rubin Tuder). In each case, the authors present a balanced overview of the field and focus on an area of particular interest, such as the contribution of excitatory neurotransmitter release to the pathogenesis of cerebral infarction, the protective effect of heat shock proteins (HSPs) in myocardial ischemia, the role of VEGF in ischemia-induced angiogenesis, the disruptive effects of ischemia on epithelial barrier function, or the effects of hypoxia on pulmonary vascular biology. Most of the research discussed employs tissue culture or small animal model systems and reflects the expectation that insights into basic pathophysiology will offer a foundation for designing therapeutics.

Despite the variability of responses to ischemia in various tissues, several general therapeutic strategies can be considered regardless of the anatomical site of ischemia. Ischemia arises when tissue demand for energy substrates (primarily $\mathrm{O}_{2}$ and glucose) is not matched by supply, usually due to impaired perfusion. Thus, ischemia can be prevented or eliminated, in principle, by decreasing demand or increasing supply. As discussed by Williams and Benjamin, decreased demand occurs in the case of hibernating myocardium, in which the ATP-consuming process of contractility is inhibited to minimize $\mathrm{O}_{2}$ and glucose consumption. Global inhibition of myocardial or cerebral function is unlikely to represent a viable therapeutic strategy, but the alternative of increasing supply to these tissues seems feasible, for example, by therapeutic angiogenesis (see Perspective by Isner). DNA- or protein-based clinical trials involving VEGF, other angiogenic factors, or mediators of their production are currently underway.

Another approach is to prevent the death of ischemic tissue, i.e., infarction. A major focus of investigation has been the preconditioning phenomena that have been demonstrated in virtually every organ, including the heart and brain. Thus, exposure of an organ or tissue to one or more brief episodes of ischemia will provide protection against subsequent prolonged ischemia that would otherwise result in infarction. The preconditioning stimulus provides an immediate but shortlived "first window" of protection, which occurs over a period of minutes to hours and requires the altered activity of pre-existing proteins, as well as a delayed but sustained "second window" of protection, which persists over a period of hours to days and depends on new protein synthesis. Considerable progress has been made in elucidating the signal transduction pathways that mediate these adaptive responses, as described in the Perspectives by Choi's group and by Williams and Benjamin. A pharmacologic agent capable of activating a preconditioning pathway would, of course, have tremendous therapeutic potential.

A third approach is to target for inhibition or induction a specific gene or protein product that is known to promote ischemia or to protect against infarction. The analysis of knockout and transgenic mice has

Ischemia arises when tissue demand for energy substrates (primarily $\mathrm{O}_{2}$ and glucose) is not matched by supply...

provided a wealth of data regarding genes that, when inactivated or activated, either promote or protect against cerebral or myocardial infarction. The interpretation of these data, however, is not always entirely straightforward. For example, the Nos 2 gene encoding inducible nitric oxide synthase is required for late-phase cardiac preconditioning, but NOS2-deficient mice develop smaller cerebral infarctions than their wild-type littermates in response to cerebral arterial occlusion. Thus, NOS2 may help to protect preconditioned animals and also, paradoxically, promote infarction in non-preconditioned animals. Transgenic models have demonstrated that overexpression of 
HSPs, such as HSP70, provides protection against ischemia in the myocardium (as described by Williams and Benjamin) and in epithelial tissues (as discussed by Nigam and colleagues). Examples of potentially useful pharmacologic inhibitors have come from animal models of hypoxia-induced pulmonary hypertension, in which treatment with angiotensin-converting enzyme inhibitors or endothelin receptor $\mathrm{ET}_{\mathrm{A}}$ antagonists can prevent or reverse vascular remodeling. However, as Voelkel and Tuder explain, it is not entirely clear to which clinical conditions the rodent model is relevant.
The present Perspective series on ischemia has notable parallels to the excellent JCI Perspective series on cancer therapy that was recently edited by Bill Kaelin (December 1999-January 2000). For both ischemic and neoplastic disorders, we are accumulating an impressive fund of knowledge regarding pathophysiology. Although the articles in the present series on ischemia demonstrate the enormous complexity of the disease processes under investigation, the challenge to each investigator and reader of the JCI remains to build this growing investment into clinical dividends, in the form of novel and effective therapeutics. 